\title{
El acceso a la educación pública de la población indígena del municipio de Nahuizalco, departamento de Sonsonate
}

\section{The access to public education in the indigenous peoples of the municipality of Nahuizalco, department of Sonsonate}

\author{
Adilia Valenzuela de Rivas' \\ David de Jesús Galdámez ${ }^{2}$ \\ Fredy Antonio Barahona-García ${ }^{3}$ \\ Henry Mauricio Alas-Rivas ${ }^{4}$ \\ Magdalena Lisbeth Beltrán-Águila ${ }^{5}$ \\ Vanessa Noemí Espinoza-Romero ${ }^{6}$ \\ Alejandra Michelle Granados-Calles ${ }^{7}$ \\ Idalia Stephanie Molina-Veliz \\ Génesis Alejandra López-Rodas 9 \\ Universidad Tecnológica de El Salvador \\ dilia.valenzuela@utec.edu.sv
}

Recibido: 21/07/17- Aceptado: 20/08/17

\section{Resumen}

El estudio analiza la forma en la que el Estado implementa políticas para el acceso a la educación en la población indígena del municipio de Nahuizalco y cómo las instituciones educativas locales utilizan mecanismos para promover la diversidad cultural y la inclusión en el ámbito educativo de los niños y las niñas pertenecientes a los pueblos indígenas de la zona; se analiza la influencia de estos aspectos en el ámbito socioeconómico y en la situación en la que se encuentran dichos pobladores. La falta de acceso a la educación de la población indígena de Nahuizalco es una problemática que con el paso del tiempo ha impedido que estos grupos puedan desarrollarse en diversos campos dentro de la sociedad. Nahuizalco es uno de los municipios en los que se concentran parte de los pueblos indígenas que hoy en día habitan en el occidente de El Salvador. La educación es un ámbito

\section{Abstract}

The manner in which the Salvadorean State implements policies for the access to education by the indigenous peoples of the municipality of Nahuizalco is analyzed in this study. It also studies how the local educational institutions use mechanisms to promote cultural diversity and inclusion in the educational environment of those boys and girls who belong to the indigenous peoples in the area. How these aspects influence the current situation of said inhabitants is also analyzed in this study. The lack of access to education that the indigenous people face has been a constant problem which has prevented them from developing and growing in different areas of society. Nahuizalco is one of the jurisdictions where Native people concentrate and live in the western part of El Salvador. Education, being a special environment, not only reflects and summarizes the abuse, discrimination

1 Catedrática de la asignatura Realidad Nacional, docente e investigadora Utec y consultora independiente en capacitación y educación.

2 Estudiante de la asignatura Realidad Nacional, Utec

3 Instructor de la asignatura Realidad Nacional, Utec.

4 Instructor de la asignatura Realidad Nacional, Utec.

5 Estudiante de la asignatura Realidad Nacional, Utec.

6 Estudiante de la asignatura Realidad Nacional, Utec

7 Estudiante de la asignatura Realidad Nacional, Utec

8 Estudiante de la asignatura Realidad Nacional, Utec

9 Estudiante de la asignatura Realidad Nacional, Utec 
especial que no solo refleja y condensa los abusos, la discriminación y marginación que tradicionalmente han sufrido las poblaciones indígenas, sino también su continua lucha por la igualdad y el respeto que siempre se les ha negado. Este estudio observa que en el actual sistema educativo de El Salvador aún no se han diseñado programas de enseñanza dirigidos a pueblos indígenas.

\section{Palabras clave}

Grupos étnicos - Educación - El Salvador; Derecho a la educación; Analfabetismo - Nahuizalco, El Salvador and marginalization that the indigenous people have suffered, but it also reflects their constant struggle for the equality and respect they have always been denied. This study points out that the current educational system of El Salvador has not yet designed learning programs aimed at the Native peoples.

\section{Keywords}

Ethnic groups - education - El Salvador - right to education - illiteracy - Nahuizalco, El Salvador

\section{Introducción}

Según el "Perfil de los pueblos indígenas en El Salvador" del Banco Mundial (BM, 2003), en el siglo XXI se encuentran distribuidos los pueblos indígenas en los departamentos de la manera siguiente: los nahua-pipiles, ubicados en Ahuachapán, Santa Ana, Sonsonate, La Libertad, San Salvador, Cuscatlán, La Paz, Chalatenango y San Vicente; los lencas, en Usulután, San Miguel, Morazán y La Unión; y los cacaoperas, en Morazán.

El estudio se realizó en el municipio de Nahuizalco, departamento de Sonsonate, en la zona occidental del país, con presencia de los pueblos nahua-pipiles. Uno de los mayores problemas que enfrenta la población indígena de este municipio es la discriminación histórica a la que ha sido expuesta; no han sido tomadas en cuenta sus necesidades, aun teniendo problemas sociales, económicos, ambientales; y han buscado que se les reivindiquen sus derechos, según el Consejo Coordinador Nacional. Indígena Salvadoreño [CCNIS] (2006). Desde la invasión y la Conquista por los españoles, los pueblos indígenas fueron sometidos; y desde la Colonia se les impuso otra cultura, otra lengua, la religión de los dominantes, otra forma de apropiación y explotación de la tierra y otra educación. Ese es el origen de muchos de los problemas que se siguen enfrentando en la actualidad. Luego de haber sido ellos los amos y los dueños de las sus tierras, ahora no tienen nada donde ellos se puedan refugiar ni mucho menos algo para heredar; no más que su cultura y las expresiones culturales que sus antepasados han dejado, como su lengua y tradiciones. Con lo antes descrito, se puede considerar que fueron problemas provocados por la dominación, la pobreza, la falta de acceso a la tierra para su cultivo y a la educación; el analfabetismo, situación que sin duda alguna aún sigue afectando a dichos grupos étnicos, tanto en ámbitos sociales, económicos, políticos como en otros aspectos que forman parte de las dimensiones en las que se desarrolla el ser humano.

Este estudio se enfoca en la educación formal y en el derecho a la educación de todos los salvadoreños. En el marco internacional de los derechos indígenas se destaca que el derecho de los pueblos indígenas a la educación está protegido por la Declaración de las Naciones Unidas sobre los Derechos de los Pueblos Indígenas, que en su artículo 14 dispone que "los pueblos indígenas tienen derecho a establecer y controlar sus sistemas e instituciones docentes que impartan educación en sus propios idiomas, en consonancia con sus métodos culturales de enseñanza y aprendizaje" (Naciones Unidas, 2016).

A pesar de lo dispuesto en estos instrumentos, en nuestro país la mayoría de los niños y las niñas en edad escolar de los pueblos indígenas tienen limitado acceso al sistema de educación formal, y por diferentes factores no pueden ejercer plenamente el derecho a la educación; y existen en materia de educación situaciones en las que no hay atención especial para los pueblos indígenas, por ejemplo, no tienen diferencias en el currículo para adecuarse a las necesidades de los pueblos indígenas; y se utilizan los mismos instrumentos y contenidos que los de la población en general, situaciones por las cuales el Estado debe velar (Naciones Unidas, 2016) 
Según Naciones Unidas (2016), en su informe "El derecho de los pueblos indígenas a la educación", menciona que

el derecho de los pueblos indígenas a la educación también está protegido por otros instrumentos internacionales de derechos humanos, como la Declaración Universal de Derechos Humanos. El Objetivo 4 de la Agenda 2030 para el Desarrollo Sostenible insta a asegurar el acceso igualitario a todos los niveles de la enseñanza y la formación profesional para las personas vulnerables, incluidas las personas con discapacidad, los pueblos indígenas y los niños en situaciones de vulnerabilidad. A pesar de lo dispuesto en estos instrumentos, la mayoría de los pueblos indígenas no pueden ejercer plenamente el derecho a la educación, y existen graves disparidades en materia de educación entre los pueblos indígenas y la población en general.

Las dificultades de acceso a la educación básica, especialmente a la media y superior, por parte de los pueblos indígenas de Nahuizalco, están condicionadas por distintos factores que les impiden a los niños, las niñas y los jóvenes tener acceso a formación académica en los niveles de bachillerato y superiores; en alguna medida, tienen mayor acceso a educación básica. Entre esos factores se encuentran la pobreza, problemas de salud, la inseguridad por maras, la división de facto del territorio dominado por las principales pandillas, la violencia de género, la criminalidad en general. Por ejemplo, el Ministerio Público de El Salvador (Marroquín, 2017) reporta 238 muertes violentas en los 28 días de febrero de 2017; y según Organización Mundial de la Salud, en un país que tiene una taza de homicidios de 10 crímenes por cada 100.000 habitantes; y en 2016 se tuvo la taza de 80 homicidios en esa proporción.

El estado salvadoreño históricamente no ha facilitado a los grupos indígenas el acceso a la educación como un medio que les permita mejorar, desarrollarse académicamente, y para acceder fácilmente al conocimiento, y que este a su vez les permita un mejor desarrollo como entes colectivos y ciudadanos capaces de triunfar económica y socialmente en nuestro país. Por el contrario, han sido reprimidos, como en los acontecimientos sociopolíticos de 1932, en tiempos del general Maximiliano Hernández Martínez; los sobrevivientes se han visto invisibilizados hasta el punto que documentos oficiales plantearon que en El Salvador no habían pueblos indígenas como en otros países de Centroamérica; y que se reconoció su existencia a partir de las luchas de sus organizaciones hasta en 2014 (UCA, 2014). Y como dice Lemus (2014), a los pueblos indígenas, en todo el período republicano en El Salvador, los derechos se les han negado por siglos.

Según el CCNIS (2003), "nuestros derechos no son un regalo de la OIT ni de las Naciones Unidas ni del gobierno. Son luchas que se han liberado a escala nacional e internacional".

Al retomar el estudio del derecho a la educación en los pueblos originarios del siglo XXI, específicamente en el municipio de Nahuizalco en Sonsonate, para dar a conocer el avance que han tenido los pueblos indígenas a raíz del reconocimiento de sus derechos. Según el último censo hecho en El Salvador, nuestra población fue de 6.2 millones de habitantes dentro de la que el $12 \%$ son pueblos originarios, los que eran y aún siguen siendo llamados "leyenda" (Montoya, 2008).

Según Lemus (2014), los pueblos originarios son nativos, los cuales conservan aún la cultura de nuestros antepasados, quienes habitaron nuestras tierras, las que hoy llamamos salvadoreñas, mucho antes de la venida de los españoles. Ellos son uno de los legados que tiene nuestra nación, y pueden ser considerados como conservadores de un patrimonio cultural. Los pueblos indígenas son organizaciones sociales que poseen, en su interior, aspectos económicos, sociales, culturales y políticos propios de sus comunidades, en otras palabras, cultura, formas de vestir, de divertirse, de religión, de trabajo, de organización y administración propias, diferentes a los de las sociedades que no son indígenas y dentro de las cuales se encuentran establecidas. En El Salvador, la población indígena que habita en la actualidad en el occidente del país está concentrado en diferentes municipios como Nahuizalco, Santo Domingo de Guzmán, Izalco y otros en el departamento de Sonsonate, y son grupos de familias y de ancianos que viven dispersos en pequeñas comunidades; la mayoría de los adultos mayores son analfabetas y viven en situación de pobreza. De manera que, para los pueblos indígenas, podemos hablar de dos tipos de derechos: el derecho consuetudinario indígena, que ha sido creado y preservado al interior de la comunidad indígena como tal; y el derecho positivo indígena, creado por un país determinado o por un grupo de países dirigido a los pueblos indígenas. 
El reflejo de la fortaleza del pueblo indígena en El Salvador lo encontramos plasmado en los saberes y conocimientos originarios, vertidos en un sistema educativo propio, que se ha visibilizado y mimetizado conscientemente en un país en donde no hay reconocimiento a su existencia (BM, 2003).

Los derechos humanos de los pueblos originarios fueron reconocidos en el año 2014 por el Órgano Legislativo salvadoreño. Los pueblos indígenas tuvieron una gran lucha, que comienza a principios de la década del 2000 cuando los mayores representantes de estos pueblos deciden apelar por sus derechos, luchar por que su cultura sea visible, protegida y difundida. Es así como deciden acercarse al CCNIS, que fue el encargado de presentar la apelación a la Comisión de Legislación y Puntos Constitucionales de la Asamblea Legislativa para que se llevara a cabo el proceso (Asamblea Legislativa, 2014).

En el Artículo 63 de la Constitución de la República se encuentra el siguiente inciso: "El Salvador reconoce a los pueblos indígenas y adoptará políticas a fin de mantener y desarrollar su identidad étnica y cultural, cosmovisión, valores y espiritualidad". La versión anterior solo decía que "la riqueza artística, histórica y arqueológica del país forma parte del tesoro cultural salvadoreño, el cual queda bajo la salvaguarda del Estado y sujeto a leyes especiales para su conservación".

Mata y Martínez (2010) hacen un buen resumen de la respuesta del Estado salvadoreño a las necesidades de los pueblos indígenas, y dicen: "Las políticas del Estado salvadoreño acerca de los/las indígenas son poco claras; este aspecto lo asume la Secretaría de Cultura a través de la Secretaría de Asuntos Indígenas creada en la década de los años noventa".

La educación, de acuerdo a la Constitución de la República, es un derecho del cual todos deben gozar, pero esta posición muchas veces excluye a los miembros de los pueblos originarios, ya que se les hace muy difícil lograrlo por diferentes factores; entre ellos es que en algunas de sus comunidades no hay escuelas cerca y se les dificulta poder asistir a una, la pobreza, la migración, y la violencia que se ha exacerbado en las zonas rurales. El sector de la educación es un ámbito especial que no solo refleja y condensa los abusos, la discriminación y marginación que tradicionalmente han sufrido nuestros indígenas, sino también su continua lucha por la igualdad y el respeto que siempre se les han negado solo por ser indígenas. (Naciones Unidas, 2016).

Los náhuahablantes actuales nacieron en las décadas de los años 1920 y 1930 y fueron la última generación que aprendió náhuat. Estos ya no transmitieron la lengua a las nuevas generaciones por diversos motivos sociales, como pérdida de prestigio de esta y la desaparición de contextos en los cuales se podía utilizar, pero especialmente por el temor a la persecución gubernamental durante los años que siguieron a la masacre de indígenas y campesinos de 1932.

Nahuizalco fue considerado como uno de los municipios con más personas analfabetas, comprendiendo edades desde los 15 hasta los 70 años de edad. Según el censo hecho en el 2007, son 8.035 personas que no saben leer ni escribir, es decir, personas excluidas al derecho de la educación; solo en el departamento de Sonsonate se cuenta con un índice de analfabetismo del $20 \%$, lo que equivale a una población de 56.921 personas analfabetas (Mined, 2010).

Según el perfil de los pueblos indígenas en El Salvador (BM, 2003), el acceso por parte de los pueblos indígenas a la educación se ve influenciado por ciertas limitantes que les impiden desarrollarse académicamente y en ámbitos sociales, dentro de las cuales se pueden mencionar las siguientes:

\section{$\checkmark$ Las condiciones económicas.}

$\checkmark$ La situación de discriminación.

$\checkmark$ Falta de personal y espacios físicos en las escuelas.

$\checkmark$ Falta de un sistema educativo culturalmente adecuado.

$\checkmark$ No se cuenta con una metodología ni con materiales adecuados para el desarrollo de una educación intercultural.

$\checkmark$ Temor de parte de los pueblos indígenas frente a los intentos de ayuda externa y a los compromisos que esta pueda implicar. Esto debido a la manipulación, represión y al despojo que han sido sometidos a lo largo de la historia.

$\checkmark$ Inadecuación del calendario escolar a los períodos de siembra y cosecha.

$\checkmark$ Considerables distancias entre las viviendas y las escuelas.

En el actual sistema educativo de El Salvador, aún no se han diseñado programas de enseñanza dirigidos a pueblos indígenas. Entre las luchas de las organizaciones indígenas está el rescate y la recuperación de la cultura náhuat. "Estamos en la lucha por la sobrevivencia de nuestros pueblos. Queremos que las próximas generaciones no 
desconozcan sus raíces ancestrales", declaró Bernal (2016), miembro del CCNIS.

Para salvaguardar, rescatar y contribuir en la conservación de las culturas que quedan en el país, se requiere que en el presente se propicie una educación integral e innovadora que no solo informe y trasmita, sino que forme, renueve; que no sea contemplativa, sino ejecutante y que permita tomar conciencia de la realidad de su tiempo y de su medio. Una educación que respete sus propias religiones, cosmovisión y costumbres (Cadpi, 2012).

\section{Métodos}

La población de estudio para la obtención de la información estuvo compuesta por 5 personas, entre ellas un representante de un centro educativo, un estudiante y 3 habitantes de la zona que fuesen parte de los pueblos indígenas, y otros miembros de la comunidad, para obtener información que sustentara el desarrollo del documento mediante un conjunto de estrategias, técnicas e instrumentos que permitieran la obtención de explicaciones veraces acerca de los hechos sociales que se pretendían analizar.

Se utilizó como base el método cualitativo, el cual surgió de la necesidad de afrontar la complejidad de los problemas de investigación planteados, que subrayan las acciones de observación, el razonamiento inductivo y el descubrimiento de nuevos conceptos, y la necesidad de enfocarlos holísticamente de forma integral, describiendo minuciosamente cada situación que se nos presentó en dicha la investigación, tomando en cuenta que este método suministró datos descriptivos de aquellos aspectos impalpables de la vida y del comportamiento del ser humano, así como de sus creencias y actitudes.

Las técnicas e instrumentos de recolección de datos fueron los siguientes:

Entrevista. Se buscó tener interacción y una buena comunicación con los sujetos investigados mediante una entrevista planificada a informantes claves, tanto de la población indígena como de los ladinos, para profundizar en la percepción sobre el derecho a la educación y la situación real. En las 4 entrevistas realizadas nos dieron su opinión sobre el desarrollo del derecho a la educación en los pueblos originarios en el siglo XXI del municipio de Nahuizalco, ayudando a identificar si el derecho se estaba cumpliendo o desarrollando de forma eficaz para los pobladores.

Visitas de campo. Se visitó el lugar, se hicieron recorridos aplicando la observación; registro fotográfico y se entabló conversación con informantes claves con quienes se aplicaron las entrevistas.

Observación etnográfica. Con el propósito de recolectar información a través de las costumbres y tradiciones, como forma de aporte a nuestra investigación, esto también nos ayudó a poder observar su forma de vida y cómo se relacionan entre ellos, cómo viven los pobladores indígenas y cuáles son sus condiciones de vida, mediante una relación directa con los habitantes pertenecientes a dichos grupos y estudiando su interacción con el medio, así como intentando interpretar el desarrollo que estos pueblos han tenido en cuanto al derecho de acceso a la educación.

\section{Resultados}

De acuerdo con las respuestas obtenidas y en relación con las preguntas enfocadas al acceso a la educación de los pueblos indígenas en el municipio de Nahuizalco, se tienen las relacionadas con el interés por parte del Mined en materia educativa para los pueblos indígenas del municipio de Nahuizalco. Al respecto algunos entrevistados (docente y estudiantes) afirmaron no haber observado interés alguno por parte del Mined en el desarrollo de la educación en los pobladores indígenas, opinando que hay cantones en el municipio en los que hay escuelas, pero que necesitan ser más equipadas con otras modalidades, como la integración de la cultura indígena, la educación básica y los bachilleratos más completos, no limitados a las opciones General y Técnico Vocacional Administrativo Contable, tanto en los cantones como en el propio centro de Nahuizalco, ya que la juventud se ve obligada a salir del municipio para buscar superarse.

En cuanto al papel que debe tomar el Estado para fomentar el desarrollo del derecho a la educación en los pobladores indígenas del municipio de Nahuizalco, los entrevistados están de acuerdo con que el Estado sí debe tomar medidas adecuadas y velar por que el derecho a la educación de los pobladores indígenas sea eficaz.

Se exploró sobre los mecanismos que implementan las instituciones de la zona para la integración de la población 
indígena y si estas toman en cuenta las condiciones socioeconómicas en las que se encuentran dichos habitantes, que expresaron que no había un mecanismo donde los pobladores indígenas puedan ser integrados en las labores estudiantiles, es decir, que no se les facilita por sus recursos socioeconómicos, incluyendo que ante la necesidad de sobrevivir ellos prefieren salir a trabajar que ir a la escuela; y para algunos jóvenes existen oportunidades en cursos que integran a la población indígena, para que esta pueda superarse, con poca capacidad para la cantidad de jóvenes de las comunidades.

Sobre la asistencia de los niños y jóvenes de origen indígena a los centros educativos de la zona, mencionan que en la actualidad existen muchas limitantes que impiden que la población indígena se desarrolle académicamente. Los siguientes son sus comentarios.

No, en lo absoluto. Esto depende de a qué distancia se encuentren estas personas; por ejemplo, hay jóvenes en cantones ubicados a kilómetros de distancia de una escuela y necesitan caminar kilómetros para llegar a dicha institución educativa (Prudencio Cruz)

A veces sí, pero depende de la situación económica en la que se encuentre cada una de las familias (Teodora Cruz)

Yo pienso que no, porque por lo general escuelas accesibles ya no hay muchas, $y$ las que hay, a nuestros hijos les quedan muy lejos (Ana Cecilia García)

Sobre la idea de que la educación es un derecho de los pobladores indígenas y la importancia que tiene, estos respondieron que el derecho a la educación es el más importante para la población, pero que existen muchas limitantes para gozar de él y por lo tanto no todos logran tener acceso a la educación y lo refieren a los escasos recursos económicos de las familias, o sea la condición de pobreza de los pueblos indígenas.

Claro que es un derecho fundamental, solamente que a muchos no se les brinda de la misma manera (Prudencio Cruz)

La educación debe formar parte de toda persona, pero hoy en día a muchos pobladores se nos dificulta poder gozar del derecho como tal (Teodora Cruz)

Se reconoce que la educación es un derecho, pero que no hay iguales oportunidades para todos los niños, las niñas y los adolescentes de las familias indígenas que están en edad escolar, por diferentes obstáculos, mayores para las familias más pobres, por lo cual algunos tienen acceso y otros no. "La educación como 'derecho' no es favorable para todos por igual, ya que no todos pueden gozar de ella, porque hay muchas cosas que se oponen para aquellos que poseen bajos recursos." (Ana Cecilia García)

Sobre las instituciones gubernamentales y su accionar para mejorar el desarrollo académico de los pobladores indígenas, los entrevistados plantean que se debe mejorar muchos aspectos en materia de educación para los pobladores indígenas. Don Prudencio Cruz hace referencia a la integración no solo de la educación formal, sino de la cultura indígena en la educación que reciben los niños y las niñas, además de dar la oportunidad de tener un aprendizaje significativo:

Lo primero que deberían hacer es facilitar el acceso a la educación en los pueblos, creando instituciones que integren a la cultura indígena, y a su vez incentivar la exigencia y el compromiso e implementar métodos para que el alumno aproveche la oportunidad que tiene para estudiar, y no aprovecharse inadecuadamente de los derechos que hoy en día existen para el joven estudiante (Prudencio Cruz).

En la misma línea de pensamiento, doña Teodora Cruz, aporta su opinión respecto a facilitar el acceso y promover la integración de la cultura indígena en el sistema de educación formal: "Hay que facilitar el acceso de la población indígena a los centros educativos, y promover en estos la integración de la cultura indígena".

Los entrevistados consideran que la educación necesita ser más equitativa, incluyendo una oferta de servicios educativos en las zonas geográficas donde no hay, y por eso los niños deben desplazarse a otros cantones o hasta la ciudad desde el área rural, afrontando el problema de la criminalidad. Tomando en cuenta la pobreza de las familias de la zona, también se deberían dar condiciones de accesibilidad 
económica, pues lo que les dan en este momento no les resuelve el problema completamente, pero se les ayuda con zapatos, uniformes, útiles y refrigerio.

Todas esas organizaciones deberían velar por una educación más equitativa, en donde se brinde lo necesario para cada uno. Tomar la iniciativa para la creación de centro educativos en donde no los hay y a su vez que cumplan con la accesibilidad económica para los pobladores indígenas (Ana Cecilia García).

\section{Discusión}

A pesar de la lucha que han tenido los pobladores indígenas del municipio de Nahuizalco, en el departamento de Sonsonate, por su derecho a la educación, todavía no se satisface con eficacia. $Y$ aunque las organizaciones gubernamentales han tomado cartas en el asunto, mediante la creación de comisiones educativas y centros escolares, estos no siempre atienden las necesidades de la población indígena.

La educación en el El Salvador, según la Constitución de la República, es un derecho del que todos pueden gozar, pero esta disposición legal en la práctica no se cumple y muchas veces excluye a algunos niños, niñas y adolescentes miembros de los pueblos originarios.

Las poblaciones indígenas necesitan oportunidades de acceso a la educación formal; y en la práctica la mayoría se ve limitada por diferentes factores socioeconómicos que les impiden acceder a un centro educativo, y la mayoría de estos no cuentan con aspectos que integren la cultura indígena.

Se observa la situación que se encuentran experimentando los pobladores indígenas, a quienes el acceso a uno de los derechos más importantes para su desarrollo se les limita cada vez más debido a diversos factores, tanto propios de las familias como de la sociedad misma. Es evidente la disparidad en materia educativa entre la población indígena y la población en general. Según los indígenas, no se toman en cuenta sus necesidades como ciudadanos de este país, y no se ha integrado la cultura indígena en ámbitos educativos y a su vez el reconocimiento de la población sobre la mínima atención por parte del Estado en el acceso de los pueblos indígenas en su derecho a la educación.
La población indígena de Nahuizalco aún no goza plenamente de su derecho a la educación, ya que, aparte de que no es muy accesible, existen factores que limitan a los pobladores en la búsqueda de alternativas para superarse, como la violencia ocasionada por los diferentes grupos delictivos, pues estos prohíben el paso en ciertas zonas, ocasionando que a los jóvenes no les sea posible asistir a alguna institución educativa. Otro factor que interfiere en esta problemática es que los habitantes tienden a pensar que el estudio es un gasto de dinero en vano debido a que hay mucho profesional sin un trabajo estable y que sea acorde con su preparación, por lo que deciden dedicarse a las labores del hogar y a la agricultura en vez de optar por superarse académicamente.

\section{Referencias}

Delors, J. (2015). La educación encierra un tesoro. Santillana. (CCNIS), C. C. (23 de junio de 2003). CCNIS: "Queremos garantizar los derechos de los pueblos indígenas". El Diario de Hoy. Recuperado el 3 de mayo de 2017 de http://www.elsalvador.com/entretenimiento/109032/ ccnis-queremos-garantizar-los-derechos-de-los-pueblos-indigenas/

Anderson , T.R. (2001). El Salvador, 1932: Ios sucesos políticos (3.a ed., Vol. 10). (J. M. Castellanos, Trad.). San Salvador, El Salvador: Dirección de Publicaciones e Impresos. Recuperado el 6 de junio de 2017 .

Banco Mundial, Unidad Regional de Asistencia Técnica (RUTA), Ministerio de Educación. Concultura y pueblos indígenas. El Salvador (2003). "Perfil de los pueblos indígenas en El Salvador". Técnico, San Salvador. Recuperado el 25 de mayo de 2017 de http://documentos. bancomundial.org/curated/es/939901468234885618/ pdf/647480PUBOPortugese0OPUBLIC0.pdf

E. Lemus, J. (23 de junio de 2014). "Los pueblos salvadoreños indígenas siempre han existido". elfaro.net. Recuperado el 2017 de https://elfaro.net/es/201406/ el_agora/15560/Los-pueblos-salvadore\%C3\%B1osind\%C3\%ADgenas-siempre-han-existido.htm

Marroquín, D. (02 de marzo de 2017). "Autoridades reportan reducción de homicidios en primeros meses de 2017". El Diario de Hoy. Recuperado el 25 de mayo de 2017 de http://www.elsalvador.com/noticias/nacional/318282/autoridades-reportan-reduccion-dehomicidios-en-primeros-meses-de-2017/ 
Mata, M., \& Martínez, Julio (3 de abril de 2010). Movimientos-sociales-indigenas-y-campesinos-resumen.

Ministerio de Salud (2009). "Política Nacional de Salud Construyendo la Esperanza". Ministerio de Salud, San Salvador. San Salvador:. Recuperado el 3 de noviembre de 2016.

Naciones Unidas (2016). “El derecho de los pueblos indígenas a la educación". Nueva York: ONU. Obtenido de http://www.un.org/es/events/indigenousday/2016/ pdf/spanish_backgrounder.pdf

STPP y Minec-Digestyc (2015). Medición multidimensional de la pobreza. El Salvador. San Salvador: Secretaría Técni- ca y de Planificación de la Presidencia y Ministerio de Economía, a través de la Dirección General de Estadística y Censos. San Salvador: Secretaría Técnica y de Planificación de la Presidencia y Ministerio de Economía a través de la Dirección General de Estadística y Censos. Recuperado en 2016 de http://www.secretariatecnica. gob.sv/wp-content/uploads/2015/10/Medici\%C3\%B3nMultidimensional-de-la-Pobreza-El-Salvador.pdf UCA (16 de junio de 2014). "Un paso histórico". Noticias UCA. Obtenido de http://www.uca.edu.sv/noticias/ texto-3004 